\title{
Geometric Construction of a Coboundary of a Cycle*
}

\author{
M. Allili ${ }^{1}$ and T. Kaczynski ${ }^{2}$ \\ ${ }^{1}$ School of Mathematics, Georgia Institute of Technology, \\ Atlanta, GA 30332-0160, USA \\ allili@math.gatech.edu \\ ${ }^{2}$ Département de Mathématiques et d'informatique, Université de Sherbrooke, \\ Sherbrooke, Québec, Canada J1K 2R1 \\ kaczyn@dmi.usherb.ca
}

\begin{abstract}
A new method of computing the homomorphism induced by a continuous map in homology presented in [1] and [2] relies on computing coboundaries of cycles. This paper is devoted to a precise geometric construction of a coboundary of a given cycle in a prescribed rectangle and a description of the associated algorithm.
\end{abstract}

\section{Introduction}

In a recent paper [2], a new method of computing the homomorphism induced by a continuous map $f$ in homology is presented. The method is based on finding a rectangularvalued point-to-set map $F$ whose graph contains the graph of $f$ and then finding a chain selector of $F$. In this approach, the computability of the homomorphism in homology depends on the ability of solving the following problem:

Given a $q$-dimensional cycle $z$ supported in a rectangular set $A$, construct a $(q+1)$ dimensional chain $c$, also supported in $A$, such that

$$
\partial c=z \text {. }
$$

Since any rectangular set $A$ is acyclic, a solution $c$ always exists. We call it a coboundary of $z$ and denote it by $\operatorname{COB}(z)$. Note that $c$ is not unique in general. Finding $c$ by direct matrix algebra methods results in a large number of algebraic operations, especially due to the fact that the involved matrices are not invertible and that computation must be done for integer coefficients, see [8]. Consequently, linear algebra leads to a high complexity of the final algorithm computing the homology of a map.

\footnotetext{
* The second author was supported by NSERC of Canada.
} 
We present, in this paper, a geometric construction which takes advantage of a rectangular form of the acyclic set $A$. The algorithm based on this method computes $C O B(z)$ in linear time with respect to the length of $z$, that is, with respect to the number of generators in the carrier of $z$. We briefly mentioned in [2] how this construction can be done but, considering its importance, it seemed to us relevant to give a precise description of the algorithm and a proof of the correctness of the formula for $C O B(z)$.

Our main motivation for studying (1.1) comes from dynamical systems where the homomorphism induced in homology by a continuous map carries important information about the dynamics of a map. We refer the reader to [2], [9], and references therein for further discussion on that topic.

Another potential motivation for studying this problem is in the fact that it is related to the problem of finding minimal surfaces of closed curves (see [3]) and, in particular, Seifert surfaces (see Chapters 4 and 5 of [13]). This is a hard and beautiful problem intensely studied by methods of differential geometry, calculus of variations, and combinatorial geometry, and we do not claim that our construction will make a miracle here. In order to adapt our construction for the study of minimal surfaces, one must generalize it for simplicial triangulations since cubical surfaces will always be far from minimal. For example, the shortest path joining two opposite vertices of a unitary square $[0,1]^{2}$ is the diagonal of length $\sqrt{2}$ whereas our algorithm in the unitary cubical grid would give a sum of two edges of total length 2. Generalizing our construction to simplicial triangulations is a work in progress. If this is done, the two problems can be related as follows:

Let $\mathcal{C}$ be a polygonal closed curve with consecutive vertices

$$
v_{0}, v_{1}, v_{2}, \ldots, v_{n-1}, v_{n}=v_{0}
$$

which approximates a given smooth curve $\mathcal{K}$. The first rough attempt would be to take a convex hull $A$ (it is an acyclic set) of all vertices of $\mathcal{C}$ and triangulate it. Then the support of the cycle

$$
z:=\left[v_{0}, v_{1}\right]+\left[v_{1}, v_{2}\right]+\cdots+\left[v_{n-1}, v_{0}\right]
$$

of the simplicial complex of $A$ is $\mathcal{C}$. If we solve (1.1), the support $\mathcal{S}$ of $c$ will be a twodimensional surface whose one-dimensional skeleton contains $\mathcal{C}$. Obviously we have no guarantee that our surface is close to a minimal surface of $\mathcal{K}$ but maybe the search for an optimal triangulation and the best solution of (1.1) can be somewhat refined.

To the authors' best knowledge, the problem (1.1) in arbitrary dimension has not been studied from the computational point of view prior to [1] and [2]. For recent results on computing homology of spaces, we refer the reader to [4], [5], [10], [12] and [18]. For an overview of problems of computational topology, refer to [6].

Since the first submission of this paper, important progress has been made in the direction of designing programs computing the homology of spaces and maps. A software based on the algorithms presented in [10] has been developed by Pilarczyk [19]. A parallel work based on [12] has been done by Kalies and Watson. An algorithm for solving (1.1) by the above discussed linear algebra methods has been found by Mazur and Szybowski [15]. The authors of that paper also wrote a computer program for the homology of a multivalued map $F$ with two options to choose: our geometric algorithm or their 
algebraic algorithm. Their experiments seem to confirm the efficiency of the geometric construction. That program is included in the Pilarczyk folder [19].

The paper is organized as follows. In Section 2 we briefly summarize preliminary definitions related to cubical grids and cubical chain complexes. A more systematic exposition can be found in [11]. In Section 3 we start from a simple example aimed at providing the reader with geometric intuitions behind the recursive formula for $\operatorname{COB}(z)$. We next state the formula and prove Theorem 3.2 which states that $\partial C O B(z)=z$ and thus it affirms the correctness of the construction. Although the construction has a clear geometric flavor, the proof of the Theorem 3.2 is not trivial and it constitutes the main achievement of this paper. In Section 4 we give some more examples illustrating the construction. In Section 5 we describe the algorithm and discuss its complexity.

\section{Basic Concepts}

The notions of grids, representable sets, and representable maps are presented in their general formulation in [16]. Representable sets and maps for convex polyhedral grids are discussed in [1] and [2]. We recall in this paper the most significant case of cubic grids in $\mathbb{R}^{n}$. The cubic grid $\mathcal{E}_{k}$ of mesh $1 / k, k=0,1,2, \ldots$, is the collection of all cubes $e \in \mathbb{R}^{n}$ of the form $e=(1 / k)\left(x+I_{1} \times I_{2} \times \ldots \times I_{n}\right)$ where $x \in \mathbb{Z}^{n}$ and $I_{j}$ is either the open interval ] 0,1 [ or the singleton $\{0\}$. We call the elements of $\mathcal{E}_{k}$ elementary cubes. Note that $\mathbb{R}^{n}$ is the disjoint union of $e \in \mathcal{E}_{k}$. There are many advantages of such decompositions over simplicial triangulations, for example, the product of two elementary cubes is an elementary cube which is not true about simplexes. A subset $X$ of $\mathbb{R}^{n}$ is said to be representable over $\mathcal{E}_{k}$ or, shortly, $k$-representable, if there exists a finite $\mathcal{E}^{\prime} \subset \mathcal{E}_{k}$ such that $X=\bigcup \mathcal{E}^{\prime}$ and $X=\bar{X}$. In particular, $X$ is a finite polyhedron. The set $\mathcal{E}^{\prime}$ is uniquely defined and denoted by $\mathcal{E}_{k}(X)$. The family of all $k$-representable polyhedra in $\mathbb{R}^{n}$ is denoted by $\mathcal{R}_{k}$.

Any cubic grid $\mathcal{E}_{k}$ can be brought to the unitary cubic grid $\mathcal{E}=\mathcal{E}_{1}$ by simple rescaling $x \mapsto k x, x \in \mathbb{R}^{n}$, which is an isomorphism of $\mathbb{R}^{n}$. This isomorphism maps the set $\mathcal{R}_{k}$ to $\mathcal{R}=\mathcal{R}_{1}$. In a similar way, multiplying by different factors on each coordinate axis permits rescaling general rectangular grids to get $\mathcal{E}$. Grid refinements are only needed for purposes of numerical approximation of sets and maps. In this paper we always work in one fixed grid, so it is enough to present the definitions and results for the unitary cubic grid $\mathcal{E}$.

Given $X \in \mathcal{R}$, the cubical chain complex of $X$ is the pair of sequences

$$
C(X)=\left(\left\{C_{q}(X)\right\}_{q \in \mathbb{Z}},\left\{\partial_{q}: C_{q}(X) \rightarrow C_{q-1}(X)\right\}_{q \in \mathbb{Z}}\right)
$$

defined as follows. Each $C_{q}(X)$ is a free abelian group generated by the set of elementary cubes $\mathcal{E}^{q}(X)$. We put $C_{q}(X)=0$ if $q<0$ or $q>n$. Thus the elements of this group, called chains, are formal linear combinations of elementary cubes. The carrier of a chain $c=\sum c_{i} e_{i}$ is the cubical set defined by

$$
|c|:=\bigcup\left\{\bar{e}_{i}: c_{i} \neq 0\right\},
$$

where $\bar{e}_{i}$ is the closure of $e_{i}$. 
The map $\partial_{q}: C_{q}(X) \rightarrow C_{q-1}(X)$, called the boundary map, is a group homomorphism defined below on elementary cubes and extended by linearity to all chains. First, for the cell $\left.I^{q}=\right] 0,1\left[{ }^{q} \subset \mathbb{R}^{q}\right.$, we put

$$
A_{i} I^{q}=\left\{\left(x_{1}, \ldots, x_{i-1}, 0, x_{i+1}, \ldots, x_{q}\right): x \in I^{q}\right\}
$$

and

$$
B_{i} I^{q}=\left\{\left(x_{1}, \ldots, x_{i-1}, 1, x_{i+1}, \ldots, x_{q}\right): x \in I^{q}\right\} .
$$

The faces $A_{i} I^{q}$ and $B_{i} I^{q}$ are called, respectively, the front $i$-face and the back $i$-face of $I^{q}$. We let

$$
\partial_{q} I^{q}=\sum_{i=1}^{q}(-1)^{i}\left(A_{i} I^{q}-B_{i} I^{q}\right) .
$$

Now let $q$-cell $e \in \mathcal{E}^{q}(X), X \subset \mathbb{R}^{n}$ be of the form $e=x+I_{1} \times I_{2} \times \cdots \times I_{n}$ where $x \in \mathbb{Z}^{n}$ and $I_{j}$ is either the open interval ]0, 1[ or the singleton $\{0\}$. The formula for $\partial_{q} e$ is more complicated than that for $I^{q}$ since some of the intervals $I_{j}$ may be singletons and, in this case, the $j$ th coordinate does not contribute to the sign alternation. It is easily seen that $e$ is the image of $I^{q}$ under the affine-linear map $T$ given by the formula

$$
\left\{T\left(y_{1}, y_{2}, \ldots, y_{n}\right)\right\}_{j}=\left\{\begin{array}{lll}
x_{j} & \text { if } & I_{j}=\{0\}, \\
x_{j}+y_{j} & \text { if } & \left.I_{j}=\right] 0,1[.
\end{array}\right.
$$

The map $T$ is composed of an insertion of zeros on chosen $(n-q)$ coordinates corresponding to the indices $j$ such that $I_{j}=\{0\}$ with the translation of the origin to the vertex $x \in \mathbb{Z}^{n}$. We put $A_{i} e=T\left(A_{i} I^{q}\right), B_{i} e=T\left(B_{i} I^{q}\right)$, and define

$$
\partial_{q} e=\sum_{i=1}^{q}(-1)^{i}\left(A_{i} e-B_{i} e\right) .
$$

Note that $\partial_{0}=0$ since $C_{-1}(X)=0$. For simplicity of notation we avoid indices and write $\partial$ for $\partial_{q}$ whenever the index is clear from the context or if the statement is true for all $q$. The most important property of the boundary map is that

$$
\partial \circ \partial=0,
$$

i.e. $\partial_{q-1} \circ \partial_{q}=0$ for all $q$. For the proof that our cubical boundary map has this property we refer to [11] and [14]. A somewhat similar construction is also given in [7].

A chain $z \in C_{q}(X)$ is called a cycle or, more precisely, a $q$-cycle if $\partial z=0$. A chain $z \in C_{q}(X)$ is called the boundary if there exists $c \in C_{q+1}(X)$ such that $\partial c=z$. The set of all $q$-cycles is the subgroup $Z_{q}:=\operatorname{ker} \partial_{q}$ of $C_{q}(X)$, while the set of all boundaries is the subgroup $B_{q}:=\operatorname{im} \partial_{q+1}$. Equation (2.2) implies that im $\partial_{q+1} \subset \operatorname{ker} \partial_{q}$ and hence the quotient group $H_{q}(X):=\operatorname{ker} \partial_{q} /$ im $\partial_{q+1}$, called the $q$ th homology group of $X$, is well defined. The homology of $X$ is the sequence $H_{*}(X):=\left\{H_{q}(X)\right\}$.

For a reader who is not familiar with homology, we should mention much information can be extracted just from the dimension $\beta_{q}$ of the free component of the group $H_{q}(X)$. This number is called the qth Betti number of $X$. Thus $\beta_{0}$ is the number of connected 
components of $X, \beta_{1}$ is the number of nontrivial fundamental (i.e. generic) loops in $X$, and, if $X \subset R^{3}, \beta_{2}$ is the number of "holes" in $X$ (think about holes in a brick of Ementaler cheese).

A representable set $X$ is called acyclic if it has the same homology as a point. The homology of a point $p$, however, is not all trivial:

$$
H_{q}(\{p\})= \begin{cases}0 & \text { if } \quad q \neq 0, \\ \mathbb{Z} & \text { if } \quad q=0 .\end{cases}
$$

In order to associate trivial homologies with acyclic spaces one introduces reduced homology $\tilde{H}_{*}(X)$ as follows: $\tilde{H}_{q}(X)=H_{q}(X)$ if $q \neq 0$ and $\tilde{H}_{q}(X)=\operatorname{ker} \varepsilon / \operatorname{im} \partial_{1}$, where the map $\varepsilon: C_{0}(X) \rightarrow \mathbb{Z}$, called the augmentation map, is given on vertices (zero-dimensional elementary cubes) by $\varepsilon v:=1$ for all $v \in \mathcal{E}_{0}(X)$.

The reduced $q$-cycles in $\tilde{Z}_{q}(X)$ are the same as cycles if $q \neq 0$ and they are elements of $\tilde{Z}_{0}(X)=\operatorname{ker} \varepsilon$ if $q=0$. It is easily seen that the reduced 0 -cycles are generated by chains $v-u$ where $u, v$ are vertices. If $X$ is acyclic, then all reduced cycles are boundaries. That implies, in particular, that any two vertices of $X$ can be connected by a path of edges. For more details see, e.g. [17].

We finish this section with the remark that any representable cubical set $X$ can be triangulated and its cubical homology defined above is isomorphic to the classical simplicial homology. Conversely, a polyhedron $P$ is not necessarily a cubical set but it is homeomorphic to one, thus the two homology theories are equivalent. For details, refer to $[11]$.

\section{Coboundary Construction}

Let then $A \subset \mathbb{R}^{n}$ be a representable rectangle, i.e. a Cartesian product of intervals with integer coordinates. Given a reduced cycle $z \in \tilde{Z}_{q}(A)$, we construct a chain $c \in C_{q+1}(A)$ such that $\partial_{q+1} c=z$. We denote this procedure by $c:=C O B(z)$ where $C O B$ stands for the word coboundary. We construct in a general manner an operator

$$
C O B: C_{q}(A) \longrightarrow C_{q+1}(A),
$$

by defining $\operatorname{COB}(\sigma)$ for all $\sigma \in C_{q}(A)$.

We start from a simple example.

Example 3.1. Consider the clockwise oriented cycle $z=e_{8}+e_{7}+e_{6}+e_{5}-e_{4}-e_{3}-$ $e_{2}-e_{1}$, in $\mathbb{R}^{2}$, where each $e_{i}$ is a positively oriented interval as indicated in Fig. 3.1. Then

$$
z \in C_{1}(R(z)) \quad \text { where } \quad R(z)=[0,2]^{2} .
$$

Let $\pi_{1}$ be the canonical projection onto the $x_{2}$-axis and let $R_{1}(z)=\{0\} \times[0,2]$ be the image of $R(z)$ under this projection. For each interval $e_{i}$ that is not projected to itself or a point, define $\left[\pi_{1}\left(e_{i}\right), e_{i}\right]$ to be the formal sum of the unit squares in which $e_{i}$ is projected down to $\pi_{1}\left(e_{i}\right)$, otherwise define $\left[\pi_{1}\left(e_{i}\right), e_{i}\right]=0$. It follows from the definition that $\left[\pi_{1}\left(e_{1}\right), e_{1}\right]=\left[\pi_{1}\left(e_{2}\right), e_{2}\right]=\left[\pi_{1}\left(e_{7}\right), e_{7}\right]=\left[\pi_{1}\left(e_{5}\right), e_{5}\right]=\left[\pi_{1}\left(e_{8}\right), e_{8}\right]=0$, $\left[\pi_{1}\left(e_{6}\right), e_{6}\right]=E_{4},\left[\pi_{1}\left(e_{4}\right), e_{4}\right]=E_{3}+E_{4}$, and $\left[\pi_{1}\left(e_{3}\right), e_{3}\right]=E_{1}+E_{2}$. 


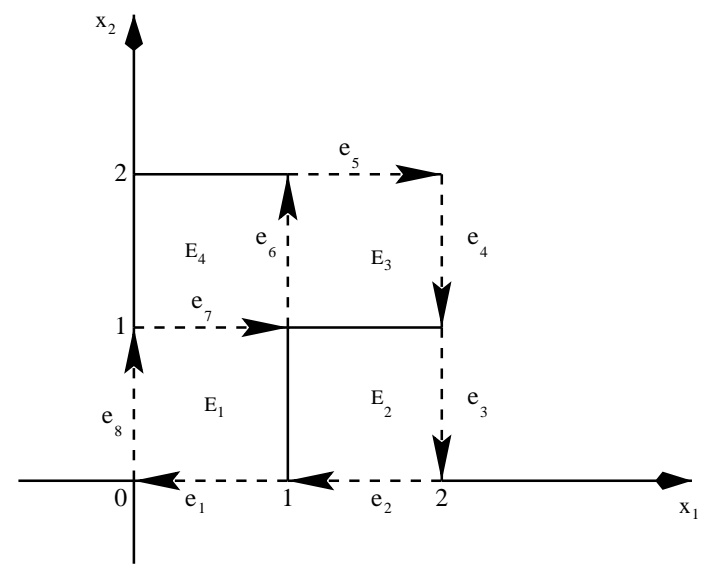

Fig. 3.1. Coboundary construction.

Now define a 2-chain $\operatorname{COB}(z)$ by replacing each $e_{i}$ in the formula of $z$ by $\left[\pi_{1}\left(e_{i}\right), e_{i}\right]$ and keeping the same coefficients, i.e.

$$
C O B(z)=0+0+E_{4}+0-E_{4}-E_{3}-E_{1}-E_{2}=-\left(E_{1}+E_{2}+E_{3}\right) .
$$

It is clearly seen that $z$ is the boundary of $\operatorname{COB}(z)$. Here, if one takes into account the orientation, then the projection of $z$ onto the $x_{2}$-axis is 0 . One asks the question whether the definition above is still correct when the projection is not trivial. Although the answer is negative, the formula above is still the main ingredient for the correct construction we provide in the rest of this section.

Let $\sigma \in C_{q}(A)$. One can write $\sigma=\sum_{i=1}^{N} c_{i} e_{i} \quad$ where $\quad e_{i} \in \mathcal{E}^{q}(A)$ and $c_{i} \neq 0, \forall i$. Each $e_{i}$ can be expressed in the form $e_{i}=\left(\left(a_{i}\right)_{1},\left(b_{i}\right)_{1}\right) \times \cdots \times\left(\left(a_{i}\right)_{n},\left(b_{i}\right)_{n}\right)$, where

$$
(a, b)=\left\{\begin{array}{lll}
] a, b[ & \text { if } & a \neq b \\
\{a\} & \text { if } & a=b .
\end{array}\right.
$$

The endpoints of intervals $\left(a_{i}\right)_{k},\left(b_{i}\right)_{k}$ are integers, either $\left(b_{i}\right)_{k}=\left(a_{i}\right)_{k}+1$ or $\left(b_{i}\right)_{k}=$ $\left(a_{i}\right)_{k}$, and the first equality holds exactly for $q$ values of $k$. Let then $R(\sigma)$ be the smallest representable rectangle in $A$ containing the carrier $|\sigma|$ of $\sigma$. It is easily seen that

$$
R(\sigma)=\left[m_{1}, M_{1}\right] \times\left[m_{2}, M_{2}\right] \times \cdots \times\left[m_{n}, M_{n}\right]
$$

where $m_{k}=\min _{i}\left\{\left(a_{i}\right)_{k}\right\}$ and $M_{k}=\max _{i}\left\{\left(b_{i}\right)_{k}\right\}$. The dimension of $R(\sigma)$ is the number of nontrivial intervals in formula (3.1).

Let $k_{1}$ be the first nontrivial coordinate of $R(\sigma)$, i.e. the smallest integer $k$ with the property $m_{k} \neq M_{k}$ (such an integer exists unless the chain $\sigma$ is trivial). Consider now the rectangle

$$
R_{k_{1}}(\sigma)=\left\{x \in R(\sigma): x_{k_{1}}=m_{k_{1}}\right\}
$$


contained in the hyperplane orthogonal to the first nontrivial direction $k_{1}$ of $R(\sigma)$. Let $\pi_{k_{1}}: R(\sigma) \longrightarrow R_{k_{1}}(\sigma)$ be the canonical projection defined by

$$
\left\{\pi_{k_{1}}(x)\right\}_{i}= \begin{cases}x_{i} & \text { if } i \neq k_{1} \\ m_{k_{1}} & \text { otherwise }\end{cases}
$$

and let $\pi_{\#}: C_{q}(R(\sigma)) \longrightarrow C_{q}\left(R_{k_{1}}(\sigma)\right)$ be the chain homomorphism induced by $\pi_{k_{1}}$. The chain homomorphism $\pi_{\#}$ is defined at level $q$ on any $q$-elementary cube $e=\left(a_{1}, b_{1}\right) \times$ $\cdots \times\left(a_{n}, b_{n}\right) \in C_{q}(R(\sigma))$ by the formula

$\pi_{\#}(e)=\left\{\begin{array}{l}0 \quad \text { if } \quad b_{k_{1}}=a_{k_{1}}+1, \\ \left(a_{1}, b_{1}\right) \times \cdots \times\left(a_{k_{1}-1}, b_{k_{1}-1}\right) \times\left\{m_{k_{1}}\right\} \times \cdots \times\left(a_{n}, b_{n}\right) \\ \text { if } \quad b_{k_{1}}=a_{k_{1}} .\end{array}\right.$

We construct, by induction with respect to the dimension $d$ of $R(\sigma)$, a $(q+1)$-chain $\operatorname{COB}(\sigma)$. Obviously the dimension of $R(\sigma)$ is at least $q$ so the induction starts from $d=q$.

For $d=q$, set $\operatorname{COB}(\sigma)=0$.

Let $d \geq q$. Suppose the construction is done for dimensions up to a certain $d \geq q$ and now let $\operatorname{dim} R(\sigma)=d+1$. For $e=\left(a_{1}, b_{1}\right) \times \cdots \times\left(a_{n}, b_{n}\right)$ in $\mathcal{E}^{q}(R(\sigma))$, we define

$\left[\pi_{\#}(e), e\right]=\left\{\begin{array}{l}0 \quad \text { if } \pi_{\#}(e)=0 \text { or } \pi_{\#}(e)=e, \\ \sum_{i=m_{k_{1}}}^{b_{k_{1}}-1}\left(a_{1}, b_{1}\right) \times \cdots \times\left(a_{k_{1}-1}, b_{k_{1}-1}\right) \times(i, i+1) \times \cdots \times\left(a_{n}, b_{n}\right) \\ \quad \text { otherwise. }\end{array}\right.$

Note that, by definition, $\left[\pi_{\#}(e), e\right]$ is a $(q+1)$-chain in $A$. If $\pi_{\#}(e) \neq 0$ and $e$ is not contained in $R_{k_{1}}(\sigma)$, then

$$
\left|\left[\pi_{\#}(e), e\right]\right|=\left(a_{1}, b_{1}\right) \times \cdots \times\left(a_{k_{1}-1}, b_{k_{1}-1}\right) \times\left(m_{k_{1}}, b_{k_{1}}\right) \times \cdots \times\left(a_{n}, b_{n}\right)
$$

is the $(q+1)$-dimensional rectangle through which $e$ is projected down to $\pi_{\#}(e)$.

The $(q+1)$-chain $\operatorname{COB}\left(\pi_{\#}(\sigma)\right)$ is well defined by induction hypotheses since $\pi_{\#}(\sigma)$ is a $q$-chain contained in $R_{k_{1}}(\sigma)$, which is a representable rectangle of dimension $d$. We then define

$$
\operatorname{COB}(\sigma):=\operatorname{COB}\left(\pi_{\#}(\sigma)\right)+\sum_{i=1}^{N} c_{i}\left[\pi_{\#}\left(e_{i}\right), e_{i}\right] .
$$

We may now state the main result of this paper.

Theorem 3.2. Let $z=\sum_{i=1}^{N} c_{i} e_{i}$ be a reduced $q$-cycle whose carrier is contained in A. Then

$$
\partial_{q+1} \operatorname{COB}(z)=z
$$

Proof. We proceed by induction on dimension $d$ of $R(z)$. As in the construction of $\operatorname{COB}(z)$, we may start the induction from $d=q$. 
If $d=q$. By definition, $\operatorname{COB}(z)=0$. On the other hand, we have $C_{q+1}(R(z))=0$, then $B_{q}(R(z))=0$ and $\tilde{Z}_{q}(R(z))=0$ since $\tilde{H}_{q}(R(z))=0$. Knowing that $z \in \tilde{Z}_{q}(R(z))$, it follows that $z=0$, and finally $\partial_{q+1} \operatorname{COB}(z)=z$.

Let $d>q$. Suppose that for $\operatorname{dim} R(z) \leq d,(3.7)$ is satisfied. Now let $\operatorname{dim} R(z)=d+1$. We begin by establishing the following result.

Lemma 3.3. We have

$$
\partial_{q+1}\left(\sum_{i=1}^{N} c_{i}\left[\pi_{\#}\left(e_{i}\right), e_{i}\right]\right)=z-\pi_{\#}(z)
$$

Proof of the Lemma. We proceed by induction with respect to the length $M=M_{k_{1}}-$ $m_{k_{1}}$ of the first nontrivial side of the rectangle $R(z)$ (which is the projection of $R(z)$ onto the $x_{k_{1}}$-axis). Note that the transition from $M$ to $M+1$ contains the arguments that prove the case $M=1$. When the length is $M+1=M_{k_{1}}-m_{k_{1}}$, the rectangle $R(z)$ is made up of $M+1$ slices, each of length 1 , along the $x_{k_{1}}$-axis. Consider

$$
R^{\prime}=\left[m_{1}, M_{1}\right] \times\left[m_{2}, M_{2}\right] \times \cdots \times\left[m_{k_{1}}, M_{k_{1}}-1\right] \times \cdots \times\left[m_{n}, M_{n}\right],
$$

the rectangle made up of the first $M$ slices of $R(z)$. Define the retraction $r: R(z) \longrightarrow R^{\prime}$ by setting, for $x \in R(z),\{r(x)\}_{i}=x_{i}$ if $i \neq k_{1}$ and

$$
\{r(x)\}_{k_{1}}= \begin{cases}x_{k_{1}} & \text { if } \quad x_{k_{1}} \leq M_{k_{1}}-1 \\ M_{k_{1}}-1 & \text { if } \quad x_{k_{1}}>M_{k_{1}}-1 .\end{cases}
$$

The chain homomorphism $r_{\#}: C_{q}(R(z)) \longrightarrow C_{q}\left(R^{\prime}\right)$ induced by $r$ at level $q$ is defined on a $q$-elementary cube $e=\left(a_{1}, b_{1}\right) \times \cdots \times\left(a_{n}, b_{n}\right) \in C_{q}(R(z))$ by the formula

$r_{\#}(e)=\left\{\begin{array}{l}0 \quad \text { if } a_{k_{1}}=M_{k_{1}}-1, b_{k_{1}}=M_{k_{1}}, \\ \left(a_{1}, b_{1}\right) \times \cdots \times\left(a_{k_{1}-1}, b_{k_{1}-1}\right) \times\left\{M_{k_{1}}-1\right\} \times \cdots \times\left(a_{n}, b_{n}\right) \quad \text { if } a_{k_{1}}=M_{k_{1}}=b_{k_{1}}, \\ e \quad \text { otherwise. }\end{array}\right.$

Set $z^{\prime}=r_{\#}(z)=\sum_{i=1}^{N} c_{i} r_{\#}\left(e_{i}\right)$, the image of $z$ by $r_{\#}$. By definition, $z^{\prime}$ is a reduced cycle in $R^{\prime}$. It is clear that for any $x \in R(z), \pi_{k_{1}}(r(x))=\pi_{k_{1}}(x)$ (see formula (3.2)) and therefore $R_{k_{1}}(z)=R_{k_{1}}\left(z^{\prime}\right)$. One can also invoke formulas (3.3) and (3.8) to check that $\pi_{\#}\left(r_{\#}(e)\right)=\pi_{\#}(e)$ for any $e \in \mathcal{E}^{q}(R(z))$. It follows that $\pi_{\#}(z)=\pi_{\#}\left(z^{\prime}\right)$.

The carrier of the reduced cycle $z-z^{\prime}$ in $C_{q}(A)$ is contained in the last slice $R^{\prime \prime}$ of $R(z)$ given by the formula

$$
R^{\prime \prime}=\left[m_{1}, M_{1}\right] \times\left[m_{2}, M_{2}\right] \times \cdots \times\left[M_{k_{1}}-1, M_{k_{1}}\right] \times \cdots \times\left[m_{n}, M_{n}\right] .
$$

At this point, we decompose each $(q+1)$-chain $\left[\pi_{\#}\left(e_{i}\right), e_{i}\right]$ as

$$
\left[\pi_{\#}\left(e_{i}\right), e_{i}\right]=\left[\pi_{\#}\left(e_{i}\right), r_{\#}\left(e_{i}\right)\right]+\left[r_{\#}\left(e_{i}\right), e_{i}\right],
$$

where $\left[\pi_{\#}\left(e_{i}\right), r_{\#}\left(e_{i}\right)\right]$ is defined as in formula (3.4) by noticing that $\pi_{\#}\left(e_{i}\right)=\pi_{\#}\left(r_{\#}\left(e_{i}\right)\right)$ and

$\left[r_{\#}\left(e_{i}\right), e_{i}\right]=\left\{\begin{array}{l}0 \quad \text { if } \quad r_{\#}(e)=0 \text { or } r_{\#}(e)=e, \\ \left(a_{1}, b_{1}\right) \times \cdots \times\left(a_{k_{1}-1}, b_{k_{1}-1}\right) \times\left(M_{k_{1}}-1, M_{k_{1}}\right) \times \cdots \times\left(a_{n}, b_{n}\right) \\ \text { otherwise. }\end{array}\right.$ 
The carrier of the $(q+1)$-chain $\Gamma=\sum_{i=1}^{N} c_{i}\left[r_{\#}\left(e_{i}\right), e_{i}\right]$ is contained in $R^{\prime \prime}$ and one can write

$$
\begin{aligned}
\sum_{i=1}^{N} c_{i}\left[\pi_{\#}\left(e_{i}\right), e_{i}\right] & =\sum_{i=1}^{N} c_{i}\left[\pi_{\#}\left(e_{i}\right), r_{\#}\left(e_{i}\right)\right]+\Gamma \\
& =\sum_{i=1}^{N} c_{i}\left[\pi_{\#}\left(r_{\#}\left(e_{i}\right)\right), r_{\#}\left(e_{i}\right)\right]+\Gamma .
\end{aligned}
$$

We first prove that

$$
\partial_{q+1} \Gamma=z-z^{\prime}
$$

This also proves the case $M=1$. Note that if $M=1$, we have $\pi_{\#}=r_{\#}, R\left(z^{\prime}\right)=R_{k_{1}}(z)$, $z^{\prime}=\pi_{\#}(z)$, and $\Gamma=\sum_{i=1}^{N} c_{i}\left[\pi_{\#}\left(e_{i}\right), e_{i}\right]$. Therefore, proving formula (3.12) will prove in case $M=1$ that

$$
\partial_{q+1}\left(\sum_{i=1}^{N} c_{i}\left[\pi_{\#}\left(e_{i}\right), e_{i}\right]\right)=z-\pi_{\#}(z) .
$$

To prove formula (3.12), it is sufficient to establish that for any cell $e \in \mathcal{E}^{q}\left(R^{\prime \prime}\right)$, the coefficient of $e$ in $z-z^{\prime}$ is equal to its coefficient in $\partial_{q+1} \Gamma$. With this in mind, we define on $C_{q}(A) \times C_{q}(A)$ a bilinear form by setting, for any two cells $e, \sigma \in \mathcal{E}^{q}(A)$,

$$
\langle e, \sigma\rangle= \begin{cases}1 & \text { if } \sigma=e, \\ 0 & \text { otherwise }\end{cases}
$$

It is then enough to verify that

$$
\left\langle e, \partial_{q+1} \Gamma\right\rangle=\left\langle e, z-z^{\prime}\right\rangle, \quad \forall e \in \mathcal{E}^{q}\left(R^{\prime \prime}\right) .
$$

Let $P_{k_{1}}$ be the projection of $R(z)$ onto the $x_{k_{1}}$-axis. We can then consider three types of $q$-cubes in $R^{\prime \prime}$ :

1. The set $\mathcal{A}$ of $q$-cubes $A$ such that $P_{k_{1}}(A)=\left\{M_{k_{1}}-1\right\}$.

2. The set $\mathcal{B}$ of $q$-cubes $B$ such that $P_{k_{1}}(B)=\left\{M_{k_{1}}\right\}$.

3. The set $\mathcal{C}$ of $q$-cubes $C$ such that $P_{k_{1}}(C)=\left(M_{k_{1}}-1, M_{k_{1}}\right)$.

The $(q+1)$-cubes in $\mathcal{E}^{q+1}\left(R^{\prime \prime}\right)$ are in one-to-one correspondence with the elements of $\mathcal{A}$ and with the elements of $\mathcal{B}$. Indeed, for each

$$
Q=\left(a_{1}, b_{1}\right) \times \cdots \times\left(M_{k_{1}}-1, M_{k_{1}}\right) \times \cdots \times\left(a_{n}, b_{n}\right),
$$

we can find a corresponding

$$
B=\left(a_{1}, b_{1}\right) \times \cdots \times\left\{M_{k_{1}}\right\} \times \cdots \times\left(a_{n}, b_{n}\right) \in \mathcal{B}
$$

and

$$
A=\left(a_{1}, b_{1}\right) \times \cdots \times\left\{M_{k_{1}}-1\right\} \times \cdots \times\left(a_{n}, b_{n}\right)=r_{\#}(B) \in \mathcal{A} .
$$

One can see that, for any $B \in \mathcal{B}$, the $q$-cube $A=r_{\#}(B) \in \mathcal{A}$. More precisely, the restriction of $r_{\#}$ to $\mathcal{B}$ is a bijection from $\mathcal{B}$ onto $\mathcal{A}$. For a given $B \in \mathcal{B}$, we denote by 
$[A, B]$ the $(q+1)$-cube obtained by projecting $B$ down to $A$. It is easily noted that if $Q \in \mathcal{E}^{q+1}\left(R^{\prime \prime}\right)$ and $B \in \mathcal{B}$ such that $Q \neq[A, B]$, then

$$
\langle B, \partial Q\rangle=\langle A, \partial Q\rangle=0 .
$$

Note that, since $B$ is a front face of $[A, B]$ and $A$ a back face of it in the sense given in the definition of $\partial$, it follows from the choice of $k_{1}$ that

$$
\langle B, \partial[A, B]\rangle=1, \quad\langle A, \partial[A, B]\rangle=-1
$$

for any any $B \in \mathcal{B}$ such that $\langle B, z\rangle \neq 0$.

We deduce from what precedes that for each $B \in \mathcal{B}$, one has

$$
\left\langle B, e_{i}\right\rangle=\left\langle[A, B],\left[r_{\#}\left(e_{i}\right), e_{i}\right]\right\rangle, \quad \forall i .
$$

Thus,

$$
\langle B, z\rangle=\langle[A, B], \Gamma\rangle=\left\langle A, z^{\prime}\right\rangle-\langle A, z\rangle, \quad \forall B \in \mathcal{B} .
$$

The latest equality is due to the fact that $A$ could be present in the formula of $z$. Thus its coefficient in $z^{\prime}$ is the sum of its coefficient in $z$ and the coefficient of $B$ in $z$ (see Fig.3.1 where the cell $e_{6}=r_{\#}\left(e_{6}\right)=r_{\#}\left(e_{4}\right)$ and therefore its coefficient in $z^{\prime}$ is 0$)$.

Then we show the identity (3.13) for elements of $\mathcal{A}, \mathcal{B}$, and $\mathcal{C}$ :

1. Let $B \in \mathcal{B}$ and $b=\left\langle B, z-z^{\prime}\right\rangle$. Since $B$ does not intersect $R^{\prime}$, it follows that $\left\langle B, z^{\prime}\right\rangle=0$. Thus $b=\langle B, z\rangle$. One can then deduce that

$$
\langle B, \partial \Gamma\rangle=b\left\langle B, \partial\left[r_{\#}(B), B\right]\right\rangle=b .
$$

2. Now let $A \in \mathcal{A}$ and $B \in \mathcal{B}$ be such that $r_{\#}(B)=A$. Let $a=\langle A, z\rangle$ and $b=\langle B, z\rangle$. By formula (3.15), we have

$$
b=\langle[A, B], \Gamma\rangle=\left\langle A, z^{\prime}\right\rangle-\langle A, z\rangle .
$$

It follows that $\left\langle A, z-z^{\prime}\right\rangle=-b$. On the other hand,

$$
\left\langle A, \partial_{q+1} \Gamma\right\rangle=b\left\langle A, \partial_{q+1}[A, B]\right\rangle=-b .
$$

3. Finally, let $C \in \mathcal{C}$ and $\alpha=\left\langle C, z-z^{\prime}\right\rangle$. By definition (see formula (3.8)), $r_{\#}(C)=0$. Therefore, $\left\langle C, z^{\prime}\right\rangle=0$, hence $\alpha=\langle C, z\rangle$. Since $\Gamma=\sum_{i=1}^{N} c_{i}\left[r_{\#}\left(e_{i}\right), e_{i}\right]$, it follows that

$$
\left\langle C, \partial_{q+1} \Gamma\right\rangle=\left\langle C, \sum_{i=1}^{N} c_{i} \partial_{q+1}\left(\left[r_{\#}\left(e_{i}\right), e_{i}\right]\right)\right\rangle=\sum_{i=1}^{N} c_{i}\left\langle C, \partial_{q+1}\left(\left[r_{\#}\left(e_{i}\right), e_{i}\right]\right)\right\rangle .
$$

Obviously, if the index $i$ is such that $\left[r_{\#}\left(e_{i}\right), e_{i}\right]=0$ or $C$ is not a face of $\left[r_{\#}\left(e_{i}\right), e_{i}\right]$, then $\left\langle C, \partial_{q+1}\left[r_{\#}\left(e_{i}\right), e_{i}\right]\right\rangle=0$. Then let $J$ be the set consisting of the indices $i \in\{0,1, \ldots, N\}$ for which $\left[r_{\#}\left(e_{i}\right), e_{i}\right] \neq 0$ and $C$ is a face of $\left[r_{\#}\left(e_{i}\right), e_{i}\right]$. For $i \in J$, denote by $\alpha_{i}=\left\langle C, \partial_{q+1}\left[r_{\#}\left(e_{i}\right), e_{i}\right]\right\rangle$ the coefficient of $C$ in $\partial_{q+1}\left(\left[r_{\#}\left(e_{i}\right), e_{i}\right]\right)$. Then

$$
\left\langle C, \partial_{q+1} \Gamma\right\rangle=\sum_{i \in J} c_{i} \alpha_{i}
$$


Let now $F$ be the $(q-1)$-face of $C$ located at the level $x_{k_{1}}=M_{k_{1}}$. It is easily seen that $F$ is a common face of $C$ and all $e_{i}, i \in J$. It follows that

$$
\begin{aligned}
0 & =\left\langle F, \partial_{q} \partial_{q+1}\left(\left[r_{\#}\left(e_{i}\right), e_{i}\right]\right)\right\rangle=\left\langle F, \partial_{q}\left(\alpha_{i} C+e_{i}\right)\right\rangle \\
& =\left\langle F, \partial_{q} e_{i}\right\rangle+\alpha_{i}\left\langle F, \partial_{q} C\right\rangle, \quad \forall i \in J .
\end{aligned}
$$

On the other hand, since $z$ is a cycle and using the precedent equality, we have

$$
0=\left\langle F, \partial_{q} z\right\rangle=\sum_{i \in J} c_{i}\left\langle F, \partial_{q} e_{i}\right\rangle+\alpha\left\langle F, \partial_{q} C\right\rangle=\left(\alpha-\sum_{i \in J} c_{i} \alpha_{i}\right)\left\langle F, \partial_{q} C\right\rangle .
$$

Hence $\alpha=\sum_{i \in J} c_{i} \alpha_{i}$, which proves formula (3.13) for elements of $\mathcal{C}$.

Up to now we proved that $\partial_{q+1} \Gamma=z-z^{\prime}$, and

$$
\partial_{q+1}\left(\sum_{i=1}^{N} c_{i}\left[\pi_{\#}\left(e_{i}\right), e_{i}\right]\right)=z-\pi_{\#}(z),
$$

for $M=1$. Let $M \geq 1$ and suppose that the length of the first nontrivial side of $R(z)$ (in the $x_{k_{1}}$ direction) is $M+1$. We recall from formula (3.11) that

$$
\sum_{i=1}^{N} c_{i}\left[\pi_{\#}\left(e_{i}\right), e_{i}\right]=\sum_{i=1}^{N} c_{i}\left[\pi_{\#}\left(r_{\#}\left(e_{i}\right)\right), r_{\#}\left(e_{i}\right)\right]+\Gamma .
$$

The smallest rectangle $R\left(z^{\prime}\right)$ containing the carrier of $z^{\prime}$ and the carrier of the $(q+1)$ chain $\sum_{i=1}^{N} c_{i}\left[\pi_{\#}\left(r_{\#}\left(e_{i}\right)\right), r_{\#}\left(e_{i}\right)\right]$ is contained in $R^{\prime}$. The first nontrivial side of $R\left(z^{\prime}\right)$ is also in the $x_{k_{1}}$ direction and is at most of length $M$. By the induction hypotheses, formula (3.12), and the fact that $\pi_{\#}(z)=\pi_{\#}\left(z^{\prime}\right)$, we can finally write

$$
\begin{aligned}
\partial_{q+1}\left(\sum_{i=1}^{N} c_{i}\left[\pi_{\#}\left(e_{i}\right), e_{i}\right]\right) & =\partial_{q+1}\left(\sum_{i=1}^{N} c_{i}\left[\pi_{\#}\left(r_{\#}\left(e_{i}\right)\right), r_{\#}\left(e_{i}\right)\right]\right)+\partial_{q+1}(\Gamma) \\
& =z^{\prime}-\pi_{\#}\left(z^{\prime}\right)+z-z^{\prime} \\
& =z-\pi_{\#}(z) .
\end{aligned}
$$

This completes the proof of the lemma.

The smallest rectangle $R\left(\pi_{\#}(z)\right)$ containing the carrier of $\pi_{\#}(z)$ is contained in $R_{k_{1}}(z)$, the rectangle obtained by projecting $R(z)$ onto the hyperplane orthogonal to the direction $x_{k_{1}}$. It follows that the dimension of $R\left(\pi_{\#}(z)\right)$ is at most $d$. Then, from the induction hypotheses and Lemma 3.3, we deduce that

$$
\begin{aligned}
\partial_{q+1}(\operatorname{COB}(z) & =\partial_{q+1}\left(\operatorname{COB}\left(\pi_{\#}(z)\right)\right)+\partial_{q+1}\left(\sum_{i=1}^{N} c_{i}\left[\pi_{\#}\left(e_{i}\right), e_{i}\right]\right) \\
& =\pi_{\#}(z)+z-\pi_{\#}(z) \\
& =z,
\end{aligned}
$$

and the theorem is now proved. 


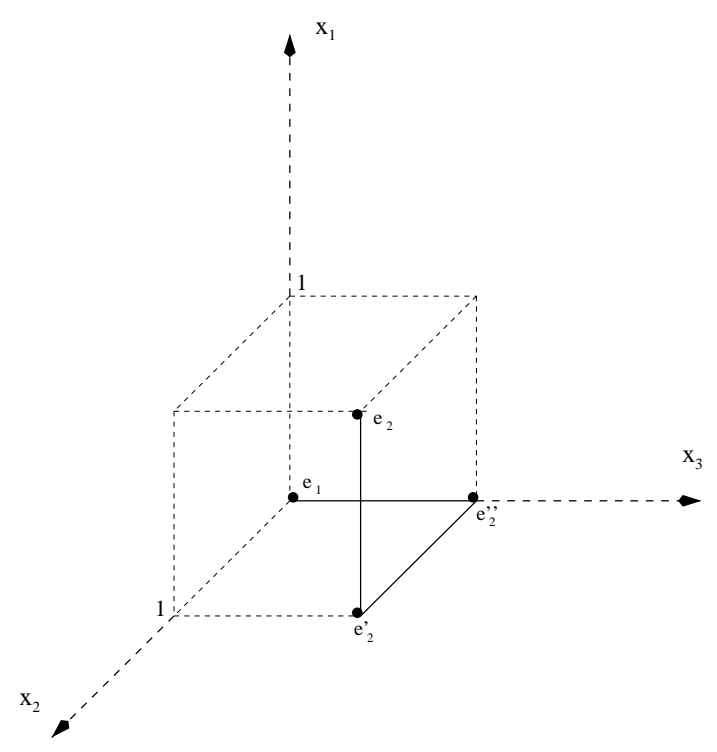

Fig. 4.1. Coboundary of a 0 -cycle in $\mathbb{R}^{3}$.

\section{Examples in $\mathbb{R}^{3}$}

The following examples provide more insight into the construction given in Section 3.

Example 4.1. Consider in $\mathbb{R}^{3}$ the reduced 0-cycle $z=e_{2}-e_{1}$, where $e_{1}=\{(0,0,0)\}$ and $e_{2}=\{(1,1,1)\}$ (Fig. 4.1). Here $R(z)=[0,1]^{3}$ is of dimension 3 and its first nontrivial direction is 1 .

Call $\pi_{1}$ the projection onto the $x_{2} x_{3}$-plane. The rectangle $R(z)$ is projected onto $R_{1}(z)=\{0\} \times[0,1]^{2}$ and $z$ is taken to the cycle $z_{1}=\left(\pi_{1}\right)_{\#}(z)=e^{\prime}{ }_{2}-e_{1}$, where $e^{\prime}=\{(0,1,1)\}$. The dimension of $R_{1}(z)$ is 2 and its first nontrivial direction is 2 . If $\pi_{2}$ is the projection onto the $x_{3}$-axis, then $R_{1}(z)$ is projected onto the rectangle $R_{2}\left(z_{1}\right)=$ $\{0\} \times\{0\} \times[0,1]$ of dimension 1 and with $x_{3}$ as the first nontrivial direction. The cycle $z_{1}$ is projected onto the cycle $z_{2}=\left(\pi_{2}\right)_{\#}\left(z_{1}\right)=e^{\prime \prime}{ }_{2}-e_{1}$, where $e^{\prime \prime}=\{(0,0,1)\}$. Finally the projection onto the origin yields the rectangle $R_{3}\left(z_{2}\right)=\{0\} \times\{0\} \times\{0\}$ of dimension 0 and $z_{2}$ is taken to the cycle $z_{3}=e_{1}-e_{1}=0$. It follows from the construction that $\operatorname{COB}\left(z_{3}\right)=0$ and therefore

$$
\begin{aligned}
\operatorname{COB}(z) & =\operatorname{COB}\left(z_{1}\right)+\left[e_{2}^{\prime}, e_{2}\right] \\
& =\operatorname{COB}\left(z_{2}\right)+\left[e^{\prime \prime}{ }_{2}, e_{2}^{\prime}\right]+\left[e_{2}^{\prime}, e_{2}\right] \\
& =\operatorname{COB}\left(z_{3}\right)+\left[e_{1}, e^{\prime \prime}{ }_{2}\right]+\left[e^{\prime \prime}{ }_{2}, e_{2}{ }_{2}\right]+\left[e_{2}^{\prime}, e_{2}\right] \\
& =\left[e_{1}, e^{\prime \prime}{ }_{2}\right]+\left[e^{\prime \prime}{ }_{2}, e_{2}^{\prime}\right]+\left[e_{2}^{\prime}, e_{2}\right] .
\end{aligned}
$$




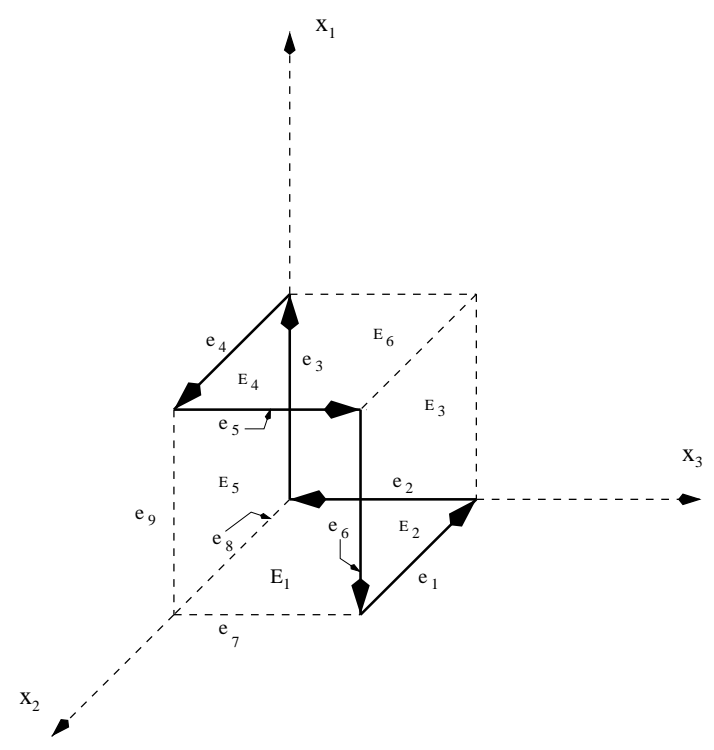

Fig. 4.2. Coboundary of a 1-cycle in $\mathbb{R}^{3}$.

Example 4.2. Consider in $\mathbb{R}^{3}$ the 1-cycle

$$
z=\sum_{i=1}^{6} c_{i} e_{i}=-e_{1}-e_{2}+e_{3}+e_{4}+e_{5}-e_{6}
$$

where each $e_{i}$ is a positively oriented interval as indicated in Fig. 4.2.

The smallest rectangle that contains the carrier of $z$ is $R(z)=[0,1]^{3}$. Its dimension is 3 and its first nontrivial direction is 1 . The projections $\pi_{1}$ and $\pi_{2}$ are defined as in the preceding example. Consider the open squares $E_{i}, i=1, \ldots, 6$, where $E_{1}$ is the base of the cube $R(z), E_{2}$ is the face having $e_{1}$ and $e_{6}$ as sides, $E_{3}$ is the face having $e_{2}$ and $e_{3}$ as sides, and $E_{6}, E_{4}, E_{5}$ are the respective opposite faces to $E_{1}, E_{2}$, and $E_{3}$. It follows that $R_{1}(z)=\{0\} \times[0,1]^{2}$. Moreover, $\pi_{1 \#}\left(e_{1}\right)=e_{1}, \pi_{1 \#}\left(e_{2}\right)=e_{2}, \pi_{1 \#}\left(e_{3}\right)=\pi_{1 \#}\left(e_{6}\right)=0$, $\pi_{1 \#}\left(e_{4}\right)=e_{8}$, and $\pi_{1 \#}\left(e_{5}\right)=e_{7}$. Finally, $\pi_{1 \#}(z)=-e_{1}-e_{2}+e_{8}+e_{7}$. It is easily seen that the projection of $\pi_{1 \#}(z)$ onto the $x_{3}$-axis is $\pi_{2 \#}\left(\pi_{1 \#}(z)\right)=0$. On the other hand, since

$$
\left[\pi_{2 \#}\left(e_{1}\right), e_{1}\right]=\left[\pi_{2 \#}\left(e_{8}\right), e_{8}\right]=\left[\pi_{2 \#}\left(e_{2}\right), e_{2}\right]=0
$$

and $\left[\pi_{2 \#}\left(e_{7}\right), e_{7}\right]=E_{1}$ we have $\operatorname{COB}\left(\pi_{1 \#}(z)\right)=E_{1}$. Finally

$$
\begin{aligned}
\operatorname{COB}(z) & =E_{1}+\sum_{i=1}^{6} c_{i}\left[\pi_{1 \#}\left(e_{i}\right), e_{i}\right] \\
& =E_{1}-0-0+0+E_{4}+E_{5}-0 \\
& =E_{1}+E_{4}+E_{5} .
\end{aligned}
$$

Remark 4.3. Obviously, there are many chains $c$ such that $\partial c$ is a given cycle. We may obtain alternative constructions by projecting $z$ onto different faces of the cube $R(z)$, 
but then the signs may change in (3.14), thus care must be taken about the alternation of sign in the formula for a boundary of a cube [2], [11].

\section{The Algorithm}

We now discuss the formal algorithm permitting the construction of a coboundary $\operatorname{COB}(z)$ for a reduced cycle $z$, namely, a chain $\operatorname{COB}(z)$ such that $\partial \operatorname{COB}(z)=z$.

Let $z=\sum_{i=1}^{N} c_{i} e_{i}$ be a $q$-reduced cycle in $\mathbb{R}^{n}$ where $0 \leq q \leq n$ and each particular $e_{i}$ is a (generator) cube of dimension $q$ in $\mathbb{R}^{n}$ that is written in the form

$$
e_{i}=\left(\left(a_{i}\right)_{1},\left(b_{i}\right)_{1}\right) \times \cdots \times\left(\left(a_{i}\right)_{n},\left(b_{i}\right)_{n}\right),
$$

where only $q$ intervals among $\left\{\left(\left(a_{i}\right)_{j},\left(b_{i}\right)_{j}\right)\right\}_{j=1}^{n}$ are nondegenerate.

The cubes will be represented by the type CUBE. The dimension of an element of type CUBE is internal to its representation. The type CHAIN will be used to represent a list of elements of type CUBE of the same dimension together with their coefficients (integers). The dimension DIM (integer) of an element of type CHAIN is the common dimension of all its elements of type CUBE. A chain is also characterized by its length LENGTH which is an integer giving the number of cubes in the chain. We do not go into the details of the definition of each of these representations here. The smallest rectangle containing $|z|$ is given by

$$
R(z)=\left[m_{1}, M_{1}\right] \times\left[m_{2}, M_{2}\right] \times \cdots \times\left[m_{n}, M_{n}\right],
$$

where $m_{k}=\min _{i}\left\{\left(a_{i}\right)_{k}\right\}$ and $M_{k}=\max _{i}\left\{\left(b_{i}\right)_{k}\right\}$. The dimension of $R(z)$ is defined to be the number of indices $i \in\{1, \ldots, n\}$ for which $m_{i} \neq M_{i}$ and is less than or equal to the dimension of the ambient space $\mathbb{R}^{n}$. The first nontrivial direction of $R(z)$ is the first index $i$ for which $m_{i} \neq M_{i}$. We therefore use the type RECTANGLE with associated dimension DIM and first nontrivial direction FIRST (necessary to define the projection) in its representation.

We can create a rectangle from a given chain $z$,

$\operatorname{RECTANGLE}(z: \operatorname{CHAIN})$;

in at most $n N$ steps where $n$ is the dimension of the ambient space $\mathbb{R}^{n}$ and $N$ is the number of cubes in the chain $z$, i.e. the length of $z$.

We make use of two functions, namely,

PROJECTION( $R:$ RECTANGLE; $e_{i}:$ CUBE): CUBE;

and

\section{$\operatorname{LIFT}\left(R:\right.$ RECTANGLE; $e_{i}:$ CUBE $):$ CHAIN;}

that are simply defined according to the definitions in formulas (3.3) and (3.5) respectively. These two functions when called run in a constant time provided the output of the function RECTANGLE is presented in an appropriate way. For more details on algorithms see [20]. 


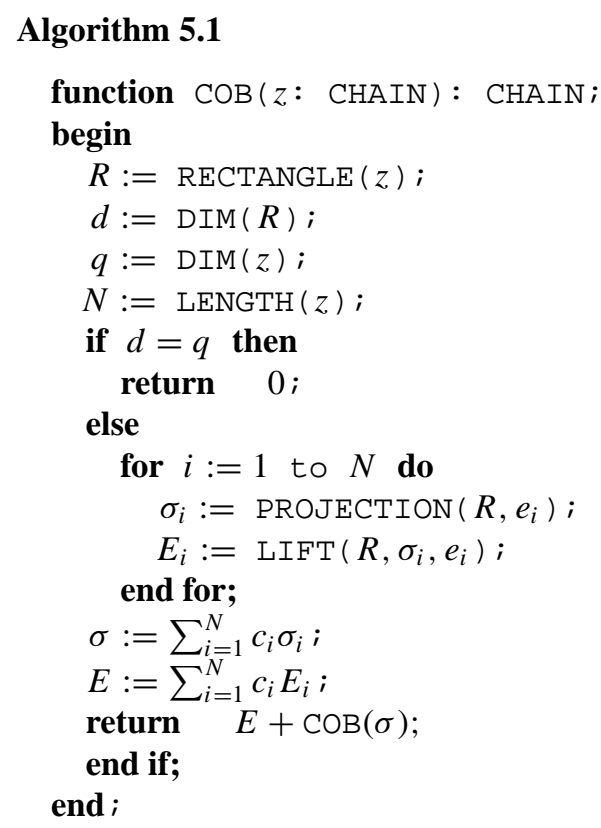

The recursive algorithm runs $d-q+1$ times, and a single call costs $O(N)$ where $N$ is the length of the cycle $z, q$ is the dimension of $z$, and $d$ is the dimension of the rectangle $R(z)$. Since we work in a fixed dimension $n$, then $d-q+1 \leq n+1$ and the algorithm runs in linear time in the number of cubes in the initial chain.

Remark 5.2. We mentioned in the Introduction, that it is possible to solve the equation $\partial c=z$ by row reduction of noninvertible matrices. When this approach is chosen, the complexity of the algorithm is about $O\left(N^{3}\right)$ in case of field coefficients and about $O\left(N^{5}\right)$ when integer coefficients are assumed.

Remark 5.3. Our construction of $c=C O B(z)$ is not minimal in the sense of the number of generators in the support of $c$. That could be improved by a judicious choice of projection faces in each step. This however will substantially increase the complexity of the algorithm.

\section{Acknowledgment}

The authors would like to thank the referees for their valuable comments and suggestions that helped enhance the presentation of this paper.

\section{References}

1. Allili, M., Une approche algorithmique pour le calcul de l'homologie de fonctions continues, Ph.D. thesis, Département de Mathématiques et d’Informatique, Université de Sherbrooke (January 1999). 
2. Allili, M., and Kaczynski, T., An algorithmic approach to the construction of homomorphisms induced by maps in homology, Trans. Amer. Math. Soc., 352 (2000), 2261-2281.

3. Dao Trong Thi and Fomenko, A.T., Minimal Sufaces, Stratified Multivarifold and the Plateau Problem, Translations of Mathematical Monographs, Vol. 84, American Mathematical Society, Providence, RI, 1991.

4. Delfinado, C.J.A., and Edelsbrunner, H., An incremental algorithm for Betti numbers of simplicial complexes on the 3-sphere, Comput. Aided Geom. Design, 12 (1995), 771-784.

5. Dey, T.K., and Guha S., Algorithms for manifolds and simplicial complexes in Euclidean 3-space, Proc. 28th ACM Sympos. Theory Comput. (1996), pp. 398-407.

6. Dey, T.K., Edelsbrunner, H., and Guha, S., Computational topology, in Advances in Discrete and Computational Geometry (eds. B. Chazelle, J. E. Goodman, and R. Pollack), pp. 109-143, South Hadley, MA (1996). Contemporary Mathematics, AMS, Providence, RI (1998).

7. Ehrenborg, R., and Hetyei, G., Generalizations of Baxter's theorem and cubical homology, J. Combin. Theory Ser. A, 69 (1995), 233-287.

8. Illiopoulos, C.S., Worst case complexity bounds on algorithms for computing the canonical structure of finite abelian groups and Hermite and Smith normal form of an integer matrix, SIAM J. Comput. 18 (1989), 658-669.

9. Kaczynski, T., Conley index for set-valued maps: from theory to computation, in Conley Index Theory (eds. K. Mischaikow, M. Mrozek, and P. Zgliczynski), pp. 57-65, Banach Center Publications, Vol. 47, Polish Academy of Science, Warsaw (1999).

10. Kaczynski, T., Mrozek, M., and Ślusarek, M., Homology computation by reduction of chain complexes, Comput. Math. Appl., 34(4) (1998), 59-70.

11. Kaczynski, T., Mischaikow, K., and Mrozek, M., Algebraic Topology: A Computational Approach (lecture notes), Preprint.

12. Kalies, D.W., Mischaikow, K., and Watson, G., Cubical approximation and computation of homology, in Conley Index Theory (eds. K. Mischaikow, M. Mrozek, and P. Zgliczynski), pp. 115-131, Banach Center Publications, Vol. 47, Polish Academy of Science, Warsaw (1999).

13. Kawauchi, A., A Survey of Knot Theory, Birkhäuser, Boston (1997).

14. Massey, W.S., A Basic Course in Algebraic Topology, Springer-Verlag, New York (1991)

15. Mazur, M., and Szybowski, J., On the Allili-Kaczynski algorithm for the construction of chain homomorphism induced by a multivalued map, Preprint.

16. Mrozek, M., An algorithmic approach to the Conley index theory, J. Dynam. Differential Equations, 11(4) (1999), 711-734.

17. Munkres, J.R., Elements of Algebraic Topology, Addison-Wesley, Reading, MA (1984).

18. Pilarczyk, P., Obliczanie Homologii Kompleksow Lańcuchowych i Zbiorów Kostkowych, M.Sc. Dissertation (in polish), Computer Science Institute, Jagellonian University, Krakow (1999).

19. Pilarczyk, P., Homology Computation-Software and Examples, http:/www.im.uj.edu.pl/ pilarczy/ homology.htm.

20. Sedgewick, R., Algorithms in C++, third edition, Addison-Wesley, Reading, MA (1998).

Received June 9, 1999, and in revised form May 10, 2000. Online publication September 22, 2000. 